\title{
Locomotion defects, together with Pins, regulates heterotrimeric G-protein signaling during Drosophila neuroblast asymmetric divisions
}

\author{
Fengwei Yu, ${ }^{1,4}$ Hongyan Wang, ${ }^{1}$ Hongliang Qian, ${ }^{1}$ Rachna Kaushik, ${ }^{2}$ Mary Bownes, ${ }^{3}$ \\ Xiaohang Yang, ${ }^{2}$ and William Chia ${ }^{1,5}$ \\ ${ }^{1}$ Temasek Lifesciences Laboratory and Department of Biological Sciences, National University of Singapore, Singapore \\ 117604; ${ }^{2}$ Institute of Molecular and Cell Biology, Proteos, Singapore 138673; ${ }^{3}$ Institute of Cell and Molecular Biology, \\ University of Edinburgh, Edinburgh, EH9 3JR United Kingdom
}

\begin{abstract}
Heterotrimeric G proteins mediate asymmetric division of Drosophila neuroblasts. Free G $\beta \gamma$ appears to be crucial for the generation of an asymmetric mitotic spindle and consequently daughter cells of distinct size. However, how $G \beta \gamma$ is released from the inactive heterotrimer remains unclear. Here we show that Locomotion defects (Loco) interacts and colocalizes with Gai and, through its GoLoco motif, acts as a guanine nucleotide dissociation inhibitor (GDI) for Gai. Simultaneous removal of the two GoLoco motif proteins, Loco and Pins, results in defects that are essentially indistinguishable from those observed in $G \beta 13 F$ or $G \gamma 1$ mutants, suggesting that Loco and Pins act synergistically to release free G $\beta \gamma$ in neuroblasts. Furthermore, the RGS domain of Loco can also accelerate the GTPase activity of Goi to regulate the equilibrium between the GDP- and the GTP-bound forms of Gai. Thus, Loco can potentially regulate heterotrimeric G-protein signaling via two distinct modes of action during Drosophila neuroblast asymmetric divisions.
\end{abstract}

[Keywords: Neuroblast; asymmetric cell division; Loco; heterotrimeric G proteins]

Received January 5, 2005; revised version accepted April 19, 2005.

Asymmetric cell division is a universal mechanism used to generate cellular diversity during development. The Drosophila embryonic central nervous system (CNS) derives largely from neural progenitors called neuroblasts (NBs). NBs delaminate from the neuroectoderm and undergo asymmetric cell division along the apical/basal axis to give rise to two daughters of distinct fate and size. The larger apical daughter cell retains a NB identity and undergoes repeated asymmetric divisions, whereas the smaller basal daughter differentiates into a ganglion mother cell (GMC) that divides only once to generate two neurons/glia (Campos-Ortega 1997). Three wellcharacterized features of the NB asymmetric divisions (Jan and Jan 2001; Knoblich 2001; Wodarz and Huttner 2003) are (1) asymmetric localization and segregation of cell fate determinants and their adaptor proteins Numb/ Partner of Numb (Pon), Prospero (Pros)/Miranda (Mira) into the basal GMC; (2) reorientation of the mitotic spindle along the apical/basal axis at metaphase; (3) gen-

Corresponding authors.

${ }^{4}$ E-MAIL fengwei@tll.org.sg; FAX 65-6-872-7007.

${ }^{5}$ E-MAIL wchia@tll.org.sg; FAX 65-6-872-7007.

Article and publication are at http://www.genesdev.org/cgi/doi/10.1101/ gad.1295505 eration of an apically biased asymmetric mitotic spindle (Kaltschmidt et al. 2000; Kaltschmidt and Brand 2002) and the displacement of the spindle toward the basal cortex during ana/telophase as well as asymmetric formation of astral microtubules (MTs) (Giansanti et al. 2001), which lead to the generation of two unequal-sized daughter cells.

These features of the NB asymmetric division are controlled by an apically localized complex of proteins that include the Drosophila homologs (Doe and Bowerman 2001) of the conserved Par3 (Bazooka, Baz)/Par6 (DmPar6)/aPKC (DaPKC) protein cassette first identified in Caenorhabditis elegans (Kemphues 2000), the novel protein Inscuteable (Insc), Gai, a subunit of heterotrimeric G proteins (Schaefer et al. 2001; Yu et al. 2003), and an evolutionarily conserved molecule, Partner of Insc (Pins) (Parmentier et al. 2000; Schaefer et al. 2000; Yu et al. 2000) that acts as a guanine nucleotide dissociation inhibitor (GDI) for Gai. Loss of single members of the apical complex, such as baz or pins, results in defective basal protein localization and spindle misorientation in mitotic NBs up to metaphase, although these defects can be partially corrected late in mitosis, a phenomenon called telophase rescue (Schober et al. 1999; Peng et al. 2000). However, unlike basal protein localization and 
spindle orientation, the generation of an asymmetric spindle and its displacement toward the basal cortex are largely unaffected, and NBs lacking one component of the apical complex usually divide like wild-type NBs to produce two unequal-sized daughter cells. Simultaneous disruption of the two redundant apical pathways, Baz/ DaPKC and Pins/G $\alpha$ i, prevents the formation of an asymmetric spindle, and two daughter cells of similar size are produced (Cai et al. 2003).

Heterotrimeric G proteins have been shown to be involved in controlling distinct microtubule-dependent processes in one-cell embryos of C. elegans (Gotta and Ahringer 2001). G $\beta \gamma$ is important for correct centrosome migration around the nucleus and spindle orientation, while G $\alpha$ subunits, GOA-1 and GPA-16, are required for asymmetric spindle positioning. Recent studies have shown that the GoLoco-motif-containing proteins, GPR1/2, act as GDIs for GOA-1 and GPA-16 to translate polarity cues, mediated by the asymmetrically localized Par proteins, into asymmetric spindle positioning in the C. elegans zygote (Colombo et al. 2003; Gotta et al. 2003; Srinivasan et al. 2003). In Drosophila NBs, heterotrimeric $G$ proteins $\mathrm{G} \beta 13 \mathrm{~F}$ and $\mathrm{G} \gamma 1$ are required for the asymmetric localization/stability of the apical components and, hence, the formation of an asymmetric spindle. This is likely to be achieved through the generation of free G $\beta \gamma$ since depletion of G $\beta \gamma$ function by overexpression of wild-type G $\alpha \mathrm{i} / \mathrm{G} \alpha \mathrm{o}$ (Schaefer et al. 2001; Yu et al. 2003) or loss of $G \beta 13 F$ or $G \gamma 1$ function (Fuse et al. 2003; Izumi et al. 2004) can lead to the generation of a symmetric and centrally placed mitotic spindle, and NBs frequently divide to produce daughter cells of similar size (henceforth referred to as "similarsized divisions,", defined below). Thus, generation of free G $\beta \gamma$ is crucial for NB asymmetric divisions. However, it is not clear whether G $\beta \gamma$ mediates spindle geometry independently of the $\mathrm{G} \alpha$ subunit(s) or alternatively by controlling the localization of $\mathrm{G} \alpha$ subunit(s) and/or the GoLoco proteins. Pins has previously been shown to act as a GDI to facilitate the dissociation of G $\beta \gamma$ from heterotrimers by binding to and stabilizing the GDPbound form of Gai (GDP-Gai) (Schaefer et al. 2001). However, paradoxically, loss of pins function does not produce the severe spindle defects seen in the $G \beta 13 F$ or G $\gamma 1$ mutant NBs, suggesting that the absence of the Pins GDI activity does not prevent the generation of free G $\beta \gamma$. Similarly, loss of Gai, while causing defects in spindle orientation and the localization of the basal proteins up to metaphase, like pins loss of function, also does not cause the severe spindle asymmetry defects seen in G $\beta 13 F$ or $G \gamma 1$ mutant NBs; however, it remains possible that additional $\mathrm{G} \alpha$ subunits may be involved in this process.

Here we show that locomotion defects (loco), a gene previously shown to be required for glial cell differentiation and dorsal-ventral patterning (Granderath et al. 1999; Pathirana et al. 2001), encodes a novel component of the NB apical complex that exhibits both guanine nucleotide dissociation inhibitor (GDI) and GTPase-activating protein (GAP) activities for Gai. Loco interacts with GDP-Gai through its GoLoco motif (Siderovski et al. 1999) and forms a complex with Gai in vivo. Loco colocalizes with Goi and Pins at the apical cortex of NBs throughout mitosis and is required for the asymmetric localization/stabilization of Pins/Gai. Analyses of various double-mutant NBs suggest that Loco, like Pins and Gai, functions redundantly with the Baz/DaPKC pathway in regulating spindle geometry. Interestingly, loss of both loco and pins functions leads to similar-sized divisions in the majority of NBs, similar to that seen in either $G \beta 13 F$ or $G \gamma 1$ mutants, suggesting that activation of $G \beta \gamma$ is mediated in a redundant manner by both Loco and Pins. Our data therefore provide functional support for the idea that the activation of heterotrimeric G-protein signaling through the generation of free G $\beta \gamma$, crucial for NB asymmetric divisions, can occur via a receptorindependent mechanism by using multiple GDIs that functionally overlap. Moreover, we show that Loco can, through its RGS domain (De Vries and Gist Farquhar 1999|, also function as a GAP to regulate the balance between GDP-G $\alpha$ i and GTP-G $\alpha$ i. Hence, both the GDI and GAP functions of Loco are important for NBs to regulate the activities of G $\alpha$ i and $\mathrm{G} \beta \gamma$.

\section{Results}

Loco, a GoLoco motif protein, interacts with GDP-Gai and can function as a GDI

In Drosophila NBs, the activation of heterotrimeric Gprotein signaling can in principle occur via a receptorindependent mechanism through the release of G $\beta \gamma$ from the inactive heterotrimer GDP-G $\alpha$ iG $\beta \gamma$, which is facilitated by the binding of Pins as a GDI to GDP-G $\alpha$ i (Schaefer et al. 2001). The GoLoco motif of Pins should therefore play a critical role through its GDI function to complex with GDP-G $\alpha$ i and generate free G $\beta \gamma$. However, previous studies have shown that inactivation of G $\beta \gamma$ by either loss of function of $G \beta 13 F$ or $G \gamma 1$ or overexpression of wild-type $\mathrm{G} \alpha \mathrm{i} / \mathrm{G} \alpha$ o leads to delocalization/ destabilization of both apical pathway components and the generation of similar-sized daughter cells in the majority of telophase NBs, whereas loss of pins function has relatively mild effects, for example, producing similarsized daughters /defined as telophase NBs from stage 10 embryos in which the ratio of the GMC/NB diameter is $\geq 0.8$; for wild-type $\mathrm{NBs}$, GMC/NB $=0.43 \pm 0.08$ ) from only a small proportion of NB divisions (15\%) (Cai et al. 2003; Fuse et al. 2003; Yu et al. 2003; Izumi et al. 2004). We reasoned that if a GDI-mediated receptor-independent mechanism were to be responsible for G-protein activation in NBs, then other unidentified GDI(s) must exist that can activate $G \beta \gamma$ activity even in the absence of pins function. We therefore searched the annotated Drosophila genome and identified only three GoLocomotif-containing proteins, namely, Pins, Loco, and RapGAP2. Further analysis indicated that while RapGAP2 appears not to be expressed in NBs (R. Kaushik, unpubl.), Loco plays a key role and is asymmetrically localized in mitotic NBs. 
There exist at least four alternatively spliced forms of Loco protein that all include a common core region containing a RGS domain, two Ras-like Raf-binding domains (RBDs), and a GoLoco motif (Fig. 3A, below). Database searches further revealed that two homologs of Drosophila Loco, RGS12 and RGS14, exist in vertebrates (Kimple et al. 2001), suggesting that loco is duplicated in vertebrates during evolution. We have confirmed a previously reported (Granderath et al. 1999) interaction between Gai and the GoLoco motif of Loco in yeast twohybrid assays. We further observed that GaiQ205L, a presumably constitutively active (GTP-bound) form, fails to interact with the GoLoco motif of Loco in yeast two-hybrid assays, suggesting that the GoLoco motif of Loco preferentially binds to GDP-Gai (Fig. 1A). These observations were further confirmed using GST pulldown assays. ${ }^{35}$ S-labeled Goi can interact with GSTGoLoco but not with GST alone, whereas ${ }^{35}$ S-labeled GaiQ205L cannot interact with GST alone and interacts very poorly with GST-GoLoco (Fig. 1B).

To show that the physical interaction between Goi and Loco reflects an in vivo interaction, we made use of a transgenic fly strain that can be induced by heat shock to express Loco-C2 fused with two tandem Flag epitopes at its $\mathrm{C}$ terminus. Loco-Flag, when induced at low levels, colocalizes with Pins and Gai as apical cortical crescents in NBs (data not shown; see also Fig. 2A-D). In coimmunoprecipitation (CoIP) experiments, when the immunocomplex was precipitated using anti-G $\alpha$ i antibody (Schaefer et al. 2001), Loco-Flag can be detected by an anti-Flag antibody, only from HS but not non-HS embryonic extracts; endogenous Pins, detected using an antiPins antibody (Yu et al. 2002), CoIPs with Gai from both HS and non-HS embryonic extracts (Fig. 1C). Although Gai can CoIP both Loco and Pins, Loco-Flag can CoIP only Gai but not Pins from HS embryonic extracts (Fig. 1D), suggesting that Loco and Pins do not simultaneously complex with the same Gai molecule. To test whether the GoLoco motifs of Loco and Pins can act as GDIs, we carried out in vitro GDI assays. The GoLoco motifs of Loco and Pins decrease the rate of exchange of GDP for GTP on G $\alpha$ i (Fig. 1E), indicating that both Pins and Loco can act as GDIs for Gai.

\section{Loco colocalizes with and depends on Pins and Gai for its apical localization}

To ascertain the subcellular localization of Loco, we generated anti-Loco antibodies against two regions of the core domain shared by all Loco isoforms (amino acids 357-636 and 564-731 of Loco-C1). These two antibodies were found to be specific for Loco since identical immunofluorescence signals were seen in wild-type embryos and these signals were absent in embryos depleted for both maternal and zygotic loco (Fig. 3P). Loco localizes as a crescent to the apical cortex as early as late interphase (Fig. 2A'). From prophase onward, Loco forms an apical crescent and segregates into the apical daughter cell at telophase (Fig. 2B'-D'), colocalizing with Pins (Fig. 2A-D) and Gai (Fig. 2E, $\mathrm{E}^{\prime}$ ) in mitotic NBs.
A

\begin{tabular}{|c|c|c|c|}
\hline & BD & BD-Goi & $\begin{array}{c}\text { BD-Gai } \\
\text { Q205L }\end{array}$ \\
\hline AD & - & - & - \\
\hline $\begin{array}{c}\text { AD- } \\
\text { Loco(GoLoco) }\end{array}$ & - & + & - \\
\hline AD-Pins & - & + & - \\
\hline
\end{tabular}

B
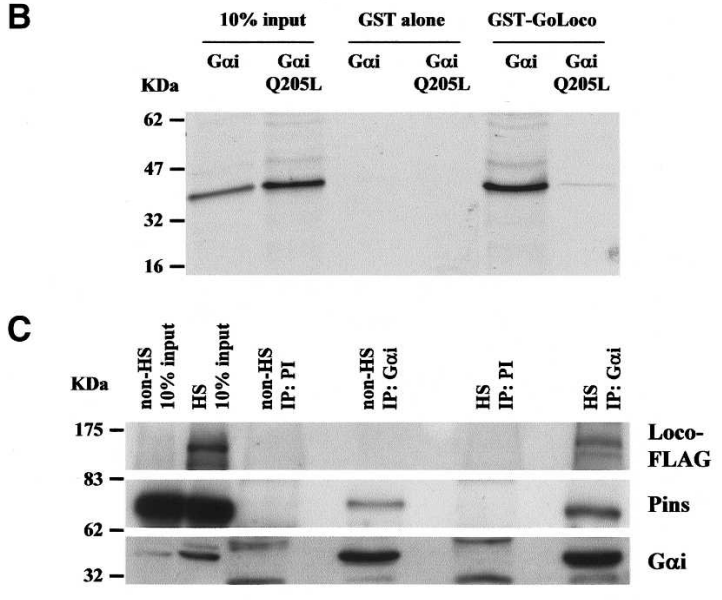

D

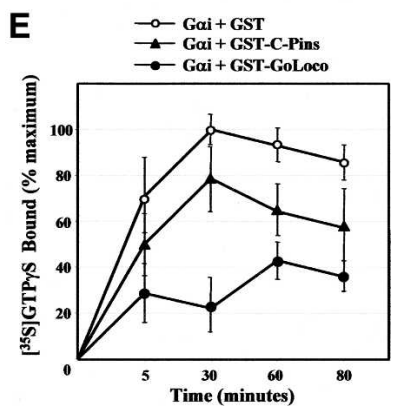

Figure 1. Loco complexes with Gai through a direct interaction and can function as a GDI for Gai. (A) The GoLoco motif of

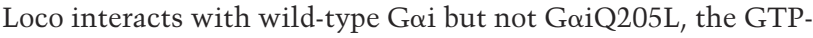
bound form of Gai, in yeast two-hybrid assays. $(+)$ Positive interaction; (-) lack of interaction. (B) In GST pull-down assays,

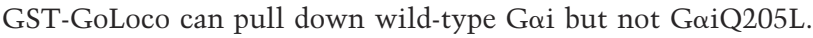
$(C, D)$ Loco and Pins can both complex with G $\alpha$ i, but not with the same Gai molecule simultaneously. Heat-shocked (HS) or non-HS embryos were collected from transgenic flies carrying hs-loco-c2. CoIP experiments were carried out using preimmune serum (PI) or anti-Gai antibody with either non-HS or HS embryo extracts. The immunocomplexes were blotted with anti-Flag, anti-Pins, and anti-G $\alpha$ i antibodies, respectively. $(C)$ Anti-Gai can pull down both Pins and Loco in vivo. $(D)$ Loco and Pins cannot be found in the same protein complex. The immunoprecipitation was carried out by using either a control IgG or anti-Flag antibody in HS embryo extracts. Loco-Flag can only pull down Gai but not Pins. (E) Both Loco and Pins possess GDI activity toward Gai. GDI assays were carried out by measuring the rate of ${ }^{35} \mathrm{~S} / \mathrm{GTP} \gamma \mathrm{S}$ binding by Gai in time-course experiments in the presence of GST alone, GST-C-Pins (amino acids 378-658), or GST-GoLoco (amino acids 564-731 of LocoC1).

To test whether asymmetric localization of Loco is dependent on other key players for NB asymmetric divisions, we examined Loco distribution in various mutants

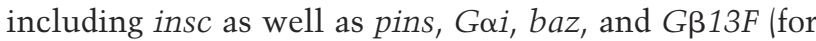


Figure 2. Loco colocalizes with Pins and Gai at the apical cortex in wild-type NBs, and its asymmetric lo-

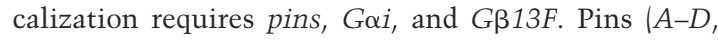
green) and Loco $\left(A^{\prime}-D^{\prime}\right.$, red) colocalize at the apical cortex in NBs from late interphase to telophase. Loco $\left(E^{\prime}\right.$, red) also colocalizes with Gai (E, green) during mitosis. In insc mutants, Loco can be observed as apical crescents with reduced intensity $(F)$, while in pins mutants, apical localization of Loco is disrupted and Loco is uniformly distributed around the cortex $(G)$. Loco is cyto-

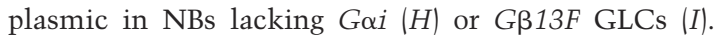
Overexpressed Gai is distributed uniformly around the cortex ( $J$, green) and causes cortical localization of Loco $\left(J^{\prime}\right.$, red). DNA is in cyan. Apical is up.

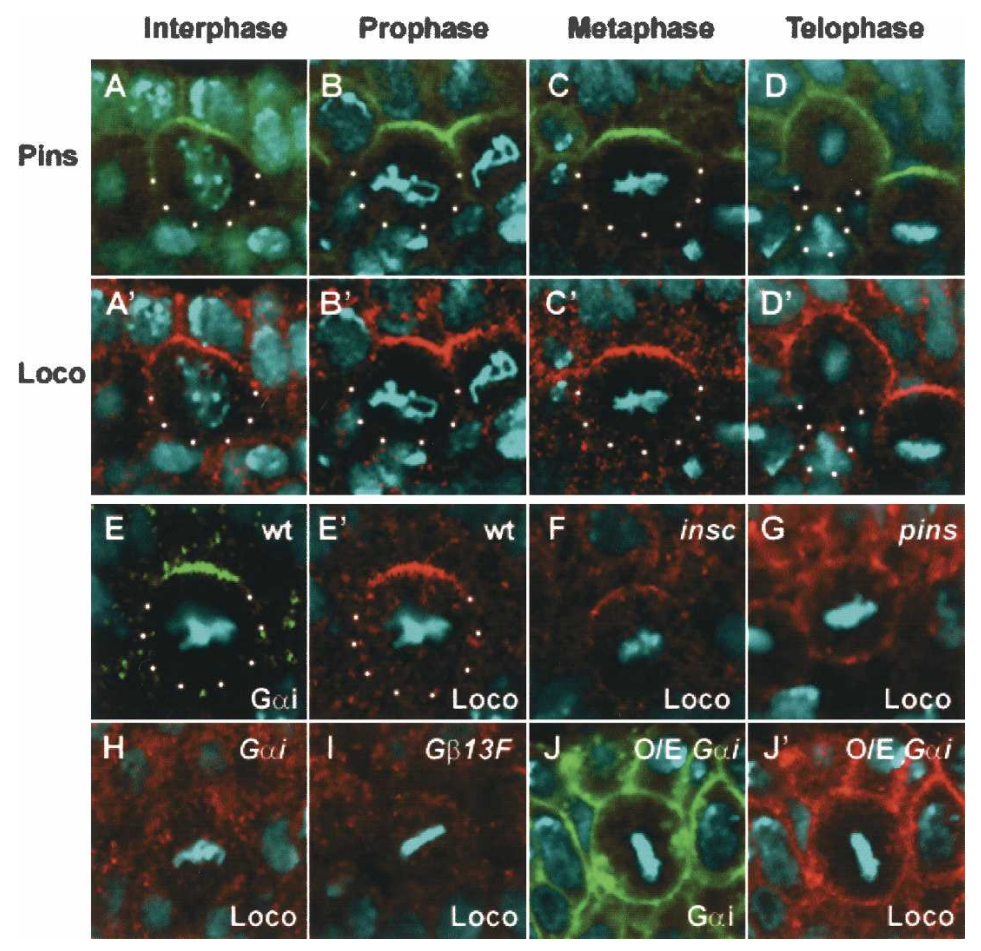

which both maternal and zygotic components were removed). In insc NBs, Loco was observed as an apical crescent of reduced intensity $(75 \%, n=48)$ (Fig. $2 \mathrm{~F}$ ) or is undetectable $(25 \%, n=48$ ) (data not shown). Similar results were seen in baz NBs (data not shown). Loco is uniformly distributed around the cortex in pins metaphase NBs (100\%, $n=20)$ (Fig. 2G); while in Gai NBs, Loco is unable to be localized to the cortex and shows cytosolic localization $(100 \%, n=29)$ (Fig. $2 \mathrm{H}$ ). Similar to that seen in Gai NBs, Loco is distributed in the cytosol with no obvious cortical signal in G $\beta 13 F$ NBs $(100 \%$, $n=32$ ) (Fig. 2I). When wild-type Gai is overexpressed, Loco (Fig. 2J') as well as Gai (Fig. 2J) and Pins (data not shown) become uniformly distributed around the cell cortex $(100 \%, n=20)$. When Insc is overexpressed in epithelial cells, Loco is recruited from the basolateral to the apical cortex (data not shown), similar to Pins (Yu et al. 2000).

Taken together, these data indicate that Loco is a novel component of the apical complex and its asymmetric localization/stability requires other apical components as well as $\mathrm{G} \beta 13 \mathrm{~F}$; its cortical localization requires Goi, and its apical localization requires Pins.

Loco is required for asymmetric localization of Gai and Pins and acts in parallel with the Baz/DaPKC pathway to mediate asymmetric daughter cell size

Given that no embryos could be obtained from germline clones (GLCs) using previously described loss-of-function alleles of loco and analyses of zygotic loss-of-function embryos revealed no obvious defects in NB asymmetric division, we carried out imprecise excisions using a P-element, EY04589, which is inserted 310 bp upstream of the start point of loco-c1 transcription (Bellen et al. 2004); three new alleles, loco $^{P 452}$, loco ${ }^{P 283}$, and ${ }_{\text {loco }}{ }^{237}$, were isolated that delete either partially or entirely the core region of the loco protein isoforms (Fig. 3A). The detailed molecular lesions associated with these alleles are given in Materials and Methods. These alleles do not show zygotic loss-of-function defects for NB divisions. Both $10 c O^{P 283}$ and $10 c O^{P 452}$ homozygotes are viable and display severe locomotion defects, similar to homozygotes of Gai and pins null mutants, suggesting that they may share similar function. To obtain loco mutant embryos that lack both maternal and zygotic components, we crossed mutant mothers homozygous for the alleles loco $^{P 283}$ or loco ${ }^{P 452}$ or trans-heterozygous for the alleles $10 c O^{P 283}$ and $10 c O^{P 237}$ to heterozygous $\operatorname{loco}^{P 283}, \operatorname{loco}^{P 452}$, or 1 loco ${ }^{P 237}$ males. Immunofluorescence confirmed that those resultant embryos are antigen-minus (Fig. 3P), suggesting that both $\operatorname{loco}^{P 283}$ and loco $^{P 452}$ are strong, possibly null alleles. Embryos derived from either 1 oco ${ }^{P 283} /$ loco $^{P 283}$ or 1 loco $^{P 283} /$ loco $^{P 237}$ mothers display indistinguishable phenotypes in NB asymmetric divisions, suggesting that $\operatorname{loco}^{P 283}$ is an amorphic allele. We henceforth refer to $10 c 0^{P 283}$ embryos lacking both maternal and zygotic components as loco mutants. In this study all phenotypic analyses described for single- and double-mutant combinations were performed using embryos lacking both maternal and zygotic components.

In the majority of loco mutant NBs, Pins is no longer apical but rather shows uniform cortical distribution with some cytosolic signal $(90 \%, n=90)$ (Fig. 3B-E). Occasionally, weak crescents of Pins were observed in in- 

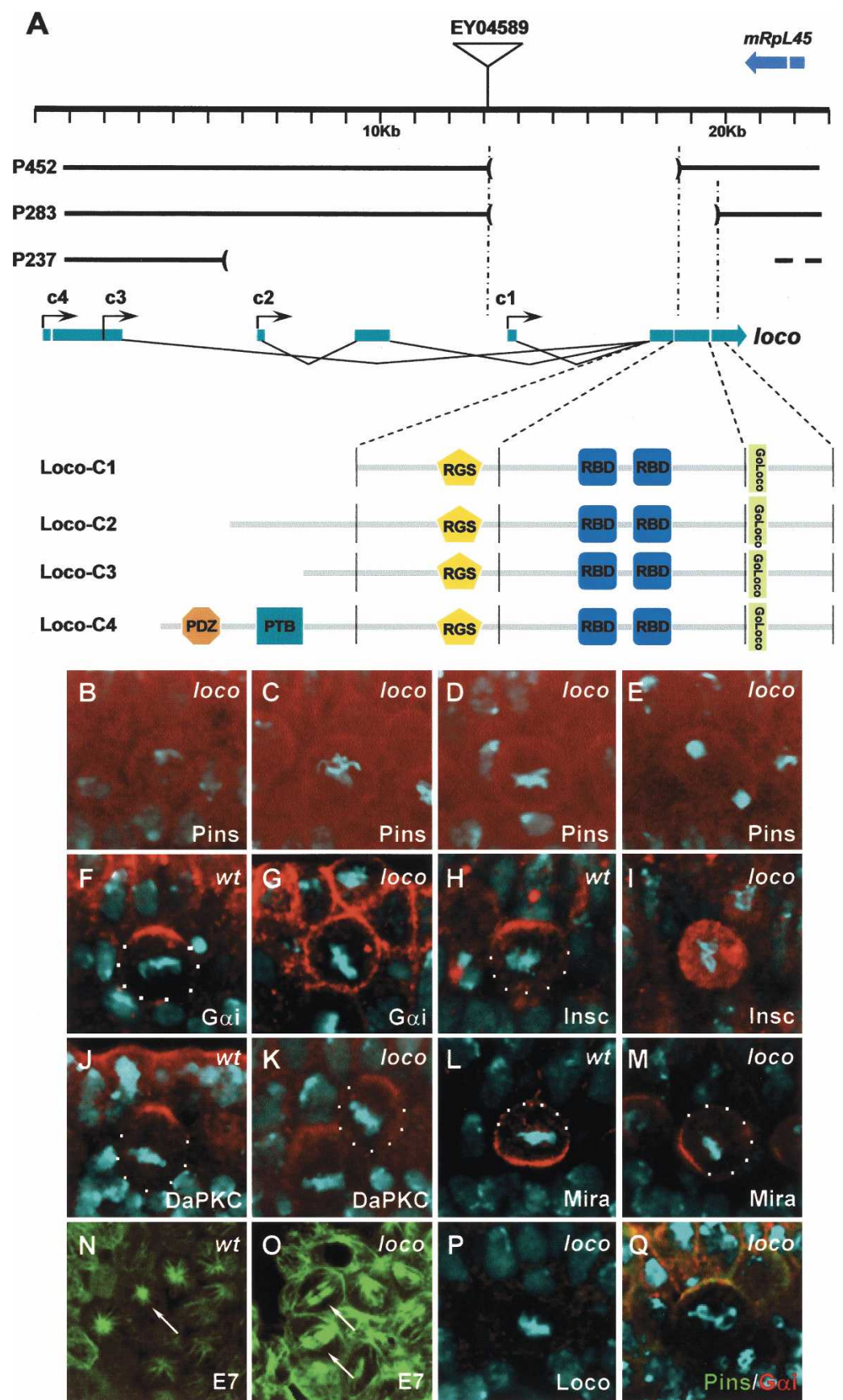

Figure 3. Loco is required for $\mathrm{NB}$ asymmetric divisions. (A) Schematic representation of four alternatively spliced forms of Loco and three loco alleles used for this study. The extent of each deletion is indicated by the parentheses. $(B-K)$ Loco is required for proper localization of other apical proteins. Pins (red) is distributed around the cell cortex and in the cytosol from interphase to telophase in loco mutants $(B-E)$. G $\alpha$ i (red), which is normally localized at the apical cortex in wild-type NBs $(F)$, is distributed uniformly around the cortex in loco mutants $(G)$. In $12 \%$ of interphase/prophase loco mutant NBs, apical Pins (green) and Gai (red) crescents could be observed with weak intensity $(Q$, showing a prophase NB). Insc (red) is apically localized in wildtype NBs $(H)$, while Insc (red) is cytosolic in loco mutants $(I)$. Compared with that seen in wildtype NBs $(J)$, DaPKC (red) remains apical in the majority of NBs, although its intensity is drastically reduced in loco mutants $(K)$. Mira (red), basally localized in wild-type NBs $(L)$, can be mislocalized in loco NBs at metaphase $(M) .(N, O)$ Spindle reorientation in cells of mitotic domain 9 is defective in loco embryos, as indicated by anti$\alpha$-tubulin staining (green). The spindle axis of wild-type domain 9 cells is perpendicular to the surface $(N)$, while in loco mutants it is often aligned parallel to the surface $(O)$. No Loco protein can be detected in loco mutant embryos $(P)$. DNA is in cyan. Apical is up in panels $B-M$. terphase/prophase NBs $(12 \%, n=43)$, where Pins colocalizes with Goi (Fig. 3Q). When detected using a specific antibody raised against full-length Gai (see Materials and Methods), Gai shows uniform cortical localization in both pins $(100 \%, n=19)$ (Schaefer et al. 2001; data not shown) and loco mutant metaphase NBs $(100 \%, n=25)$ (Fig. 3G); Insc is cytoplasmic $167 \%$, $n=45)$ (Fig. 3I); DaPKC (86\%, $n=50)$ (Fig. 3K) and Baz (data not shown) remain asymmetrically localized in the majority of loco mutant NBs, although the intensity of the crescents was dramatically reduced, a phenotype also

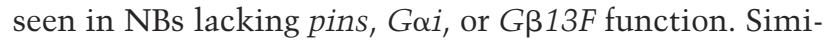

lar to that seen in pins or Gai mutants, in loco mutants the basal proteins Mira/Pros and Pon/Numb can be mislocalized relative to the overlying ectoderm at metaphase $(52 \%, n=21)$ (Fig. $3 \mathrm{M}$; data not shown). G $\beta 13 \mathrm{~F}$ remains uniformly cortical, similar to that seen in wildtype NBs (data not shown). Mitotic spindle orientation is also disturbed in loco mutants; in cells of mitotic domain 9, mitotic spindle that normally rotates by $90^{\circ}$ to align along the apical/basal axis in wild type (Fig. $3 \mathrm{~N}$ ) often fails to reorientate (Fig. 3O).

Wild-type NBs normally divide to give rise to a large apical NB and a smaller basal GMC (Fig. 4A,E). The great 


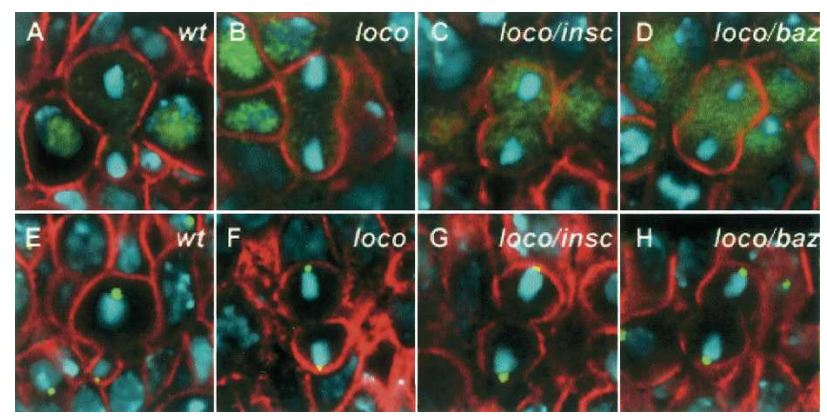

Figure 4. Loco acts redundantly with the Baz/DaPKC/DmPar6/Insc pathway to regulate spindle displacement and asymmetry, as well as daughter cell size difference. In wild-type telophase NBs $(A, E)$, the mitotic spindle (deduced from positions of the centrosomes) $(E)$ is apically biased and spindle displacement occurs toward the basal cortex to give rise to two daughter cells of unequal size. In loco mutants $(B, F), 10 \%$ of telophase NBs generate two daughter cells of similar size. loco/insc doublemutant NBs $(C, G)$ show similar-sized divisions in all telophase NBs $(100 \%$, see text). $(D, H)$ Similarly, removal of baz function in loco NBs increases the frequency of similar-sized divisions to full expressivity. $(F-H)$ In similar-sized divisions, the mitotic spindle is symmetric and both centrosomes lie in close vicinity of the cell cortex. NBs were marked by Asense, which is cytosolic green in $A-D$; BP106, a plasma membrane marker, in red $(A-H)$; and $\mathrm{CNN}$, a centrosome marker in green in $E-H$. DNA is in cyan. Apical is up.

majority of loco mutant NBs divide asymmetrically to produce daughters of different size like wild-type NBs (data not shown). However, similar to pins or Gai mutants, a small proportion of loco mutant NBs undergo similar-sized division $(10 \%, n=69)$ (Fig. 4B,F). Previous studies have suggested that two redundant pathways, the Pins/Goi and the Baz/DaPKC/(DmPar6/Insc) pathways, act redundantly to control daughter cell size difference (Cai et al. 2003). We analyzed the relative size of the two daughter cells in double mutants of loco/insc or loco/baz RNAi. In all dividing NBs, similar-sized divisions were observed in loco/insc $(100 \%, n=42)$ (Fig. 4C,G) and loco/ baz RNAi (97\%, $n=31$ ) (Fig. 4D,H) double mutants. In addition, spindle displacement and asymmetry are both disrupted in these double mutants, as revealed by anticentrosomin (CNN) staining (Fig. 4F-H).

Taken together, loco loss of function displays defects similar to those seen in pins or Gai mutants, and Loco acts redundantly with the Baz/DaPKC pathway to regulate spindle displacement and asymmetry, as well as daughter cell size difference.

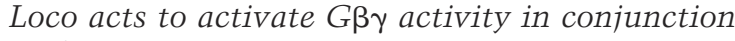
with Pins

Given that the frequency of similar-sized divisions in pins mutants is much lower than that observed in GLCs of either $G \beta 13 F$ or $G \gamma 1$ (Cai et al. 2003; Fuse et al. 2003; Izumi et al. 2004), we hypothesized the existence of an additional molecule with GDI activity that could activate G $\beta \gamma$ signaling in the absence of Pins. Loco is an obvious candidate for this role, given its function as a GDI for Gai and its role in NB division. To test our hypothesis, we generated embryos derived from double GLCs of loco and pins and compared their phenotypes with those of Gß13F GLCs. In double GLCs of pins and loco, the majority of NBs undergo symmetric divisions to generate two similar-sized daughter cells in stage 10 mutant embryos $(60 \%, n=73)$ (Fig. 5B); the cleavage plane is placed near the middle of the two centrosomes and the spindle is positioned symmetrically with both centrosomes lying in close proximity to the cell cortex (Fig. 5D), as revealed by anti-Centrosomin (CNN) staining, suggesting that spindle displacement and asymmetry are frequently disrupted in telophase NBs in the absence of both pins and loco. Astral microtubules, which are normally associated only with the apical centrosome in wild-type NBs (Fig. 5E), can emanate from both centrosomes in loco/pins double mutant NBs (Fig. 5F). These defects are strikingly similar to those observed in Gß13F mutants (Fuse et al. 2003; Yu et al. 2003), suggesting that free $\mathrm{G} \beta \gamma$ might be depleted by excessive GDP-Gai around the NB cortex when both GDIs are removed simultaneously. Consistent with this, in double GLCs of pins and loco, Gai shows uniform cortical localization in mitotic NBs $(100 \%, n=30)$ (Fig. $5 \mathrm{H}$, cf. wild type in G), colocalizing with G $\beta 13 \mathrm{~F}$ (data not shown). In loco/pins double-mutant NBs, DaPKC is either nearly undetectable in most NBs $(71 \%, n=31)$ (Fig. 5J) or shows some degree of asymmetric localization on the cell cortex in NBs when it is detectable (Fig. 5K), similar to that seen in Gß13F mutants (Fuse et al. 2003; Yu et al. 2003). Miranda is mislocalized (Fig. 5M) or delocalized (Fig. 5N) in a minority of metaphase NBs $(40 \%, n=40)$, but nevertheless segregates exclusively to one of the daughter cells during telophase in the great majority of loco/pins mutant NBs (Fig. 5P), suggesting that, similar to $G \beta 13 F$ NBs, the Baz/DaPKC function is not totally lost in loco/ pins mutant NBs.

These data indicate that maternal and zygotic depletion of both loco and pins produce phenotypes that share all of the features seen in the loss of G $\beta \gamma$ function. A detailed quantitation of the relative sizes of the $\mathrm{NB}$ daughters for various mutants further supports this view (Fig. 5Q). Our data suggest that Loco and Pins have overlapping functions as GDIs to release free $G \beta \gamma$, which, in turn, initiates downstream signaling.

\section{Ectopic expression of Loco can drive Pins off the apical cortex}

To ascertain the effects of overexpressing Loco on NB asymmetric divisions, we expressed the Loco-C1 isoform under the control of a strong maternal driver, mata-gal4 VP16 V32. Under these conditions, anti-Loco immunofluorescence in NBs appears more intense than in wild type (Fig. 6C); two types of Loco distribution were observed, uniformly cortical $(25 \%, n=64)$ (Fig. 6A) or apically enriched $(75 \%, n=64)$ (Fig. 6B). In either case, Loco colocalizes with Gai in mitotic NBs (Fig. 6A', B'). Strikingly, ectopic expression of Loco leads to cytoplasmic 


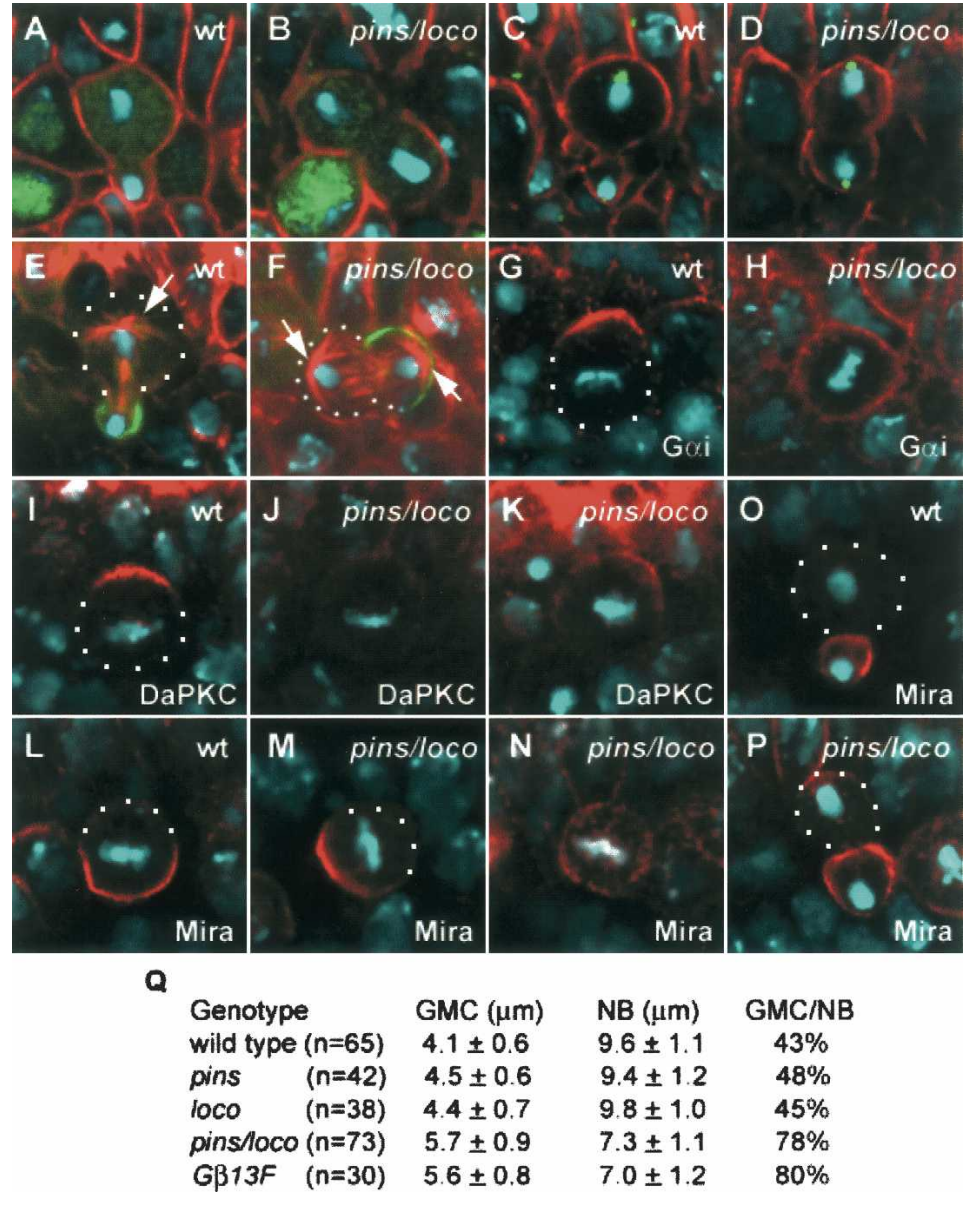

Figure 5. Loco acts to activate G $\beta \gamma$ activity in conjunction with Pins. $(A-F)$ Confocal images of triple-labeled telophase NBs (BP106, a membrane marker, red $[A-D]$; DNA, cyan $[A-F]$; Asense, a NB marker, cytosolic green $[A, B] ; C N N$, a centrosome marker, green $[C, D] ; \alpha$-tubulin, red $[E, F] ;$ and Miranda, green $[E, F] \mid$ showing unequal size divisions in wild-type $(A, C, E)$ and similar-sized divisions in loco/pins double mutants $(B, D, F)$. NBs from pins/loco double GLC embryos show high frequencies of similar-sized divisions $(B)(63 \%$, see text) in which the mitotic spindle is symmetric, as judged from $C N N$ staining (cf. wild-type $[C]$ and double mutants $[D]) .(E)$ In wild-type NBs astral microtubules are associated only with the apical centrosome; they grow out robustly and form a prominent, cap-like structure (arrow). (F) However, in loco/pins NBs that undergo similar-sized divisions, two astral microtubule caps are formed, one over each centrosome (arrows). Gai, apical in wild-type NBs $(G)$, is cortically localized in pins/loco double mutants $(H)$. In loco/pins double mutants, DaPKC is nearly undetectable in $71 \%$ of NBs $(J)$ and show weak crescents in the rest of the NBs $(K)$, compared with that in wild-type NBs $(I)$. Forty percent of loco/pins NBs $(n=40)$ show mislocalization $(M)$ or cortical localization $(N)$ of Mira at metaphase; however, as in wild type $(O)$, Mira segregates to one of the daughter cells at telophase $(P)$. DNA is in cyan. Apical is up. (Q) Quantitation of the daughter cell sizes and their ratios in wild-type and various mutant NBs. $n$ is the number of telophase NBs scored. The diameters of GMCs (the relatively smaller cell) and NBs (the relatively large cell) in telophase NBs of stage 10 embryos were measured for each genotype. The data are means \pm SD. GMC/NB is the ratio of the diameter of GMC relative to its sibling NB. distribution of Pins in the great majority of NBs $198 \%$, $n=46$ ) (Fig. $\left.6 \mathrm{D}^{\prime}\right)$, while Insc becomes primarily cytoplasmic $(100 \%, n=34)$ (Fig. $\left.6 \mathrm{E}^{\prime}\right)$, although faint cortical crescents can be seen occasionally; DaPKC localizes asymmetrically on the metaphase NB cortex $(74 \%, n=34)$ (Fig. $6 \mathrm{~F}, \mathrm{~F}^{\prime}$ ), but the crescents are broader and less intense compared with wild type; Miranda still asymmetrically localizes and segregates $(100 \%, n=20$ of telophase NBs) (data not shown). We have previously shown that Pins cortical localization depends on its association with Gai (Yu et al. 2002, 2003). The above observations are consistent with the view that excessive levels of Loco can compromise the ability of Pins to localize to the cortex by limiting the availability of GDP-Gai (see below).

Loco can act as a GAP to regulate the GTPase activity of Gai through its RGS domain

To determine whether the RGS domain of Loco is able to interact with Gai and whether this interaction is nucleotide-dependent, bacterially expressed GST or GST-RGS was incubated with in vitro translated ${ }^{35} \mathrm{~S}$-labeled Goi in the presence of GTP $\gamma \mathrm{S}$, GDP, or GDP + $\mathrm{AlF}_{4}{ }^{-}$to mimic the transition state of GTP hydrolysis. While GST-RGS is able to pull down Gai only to a low extent in the presence of either GDP or GTP $y$, the presence of
$\mathrm{GDP}+\mathrm{AlF}_{4}{ }^{-}$strongly promotes the interaction between GST-RGS and Gai (Fig. 7A, upper panel). These results suggest that the RGS domain of Loco possesses preferential affinity to the transition-state conformation of G $\alpha$ i during GTP hydrolysis. To ascertain that GST-RGS can interact with endogenous Gai from embryos, GSTRGS or GST alone was incubated with embryonic extracts. A significant amount of G $\alpha$ i could be detected by immunoblotting the protein complex bound to GSTRGS, but not in the control (Fig. 7A, lower panel), suggesting that the RGS motif of Loco is likely to interact with Gai in vivo. Since the RGS domain is able to interact with Gai, we further carried out GAP assays to test whether the RGS domain can stimulate GTP hydrolysis. In the absence of GST-RGS, Gai has only weak intrinsic GTPase activity; addition of GST-RGS fusion protein accelerates the GTPase activity of Goi significantly (Fig. $7 \mathrm{~B})$. Taken together, these data indicate that Loco can also act as a GAP for G $\alpha$ i through its RGS domain, which may, in turn, contribute to the regulation of the balance between GTP-G $\alpha$ i and GDP-G $\alpha$ i levels in NBs.

The effects of disturbing the balance of GTP-Gai and GDP-Gai on NB asymmetric divisions

To assess the effects of shifting the equilibrium of $G \alpha i$ toward either the GTP- or GDP-bound forms on NB 
Yu et al.

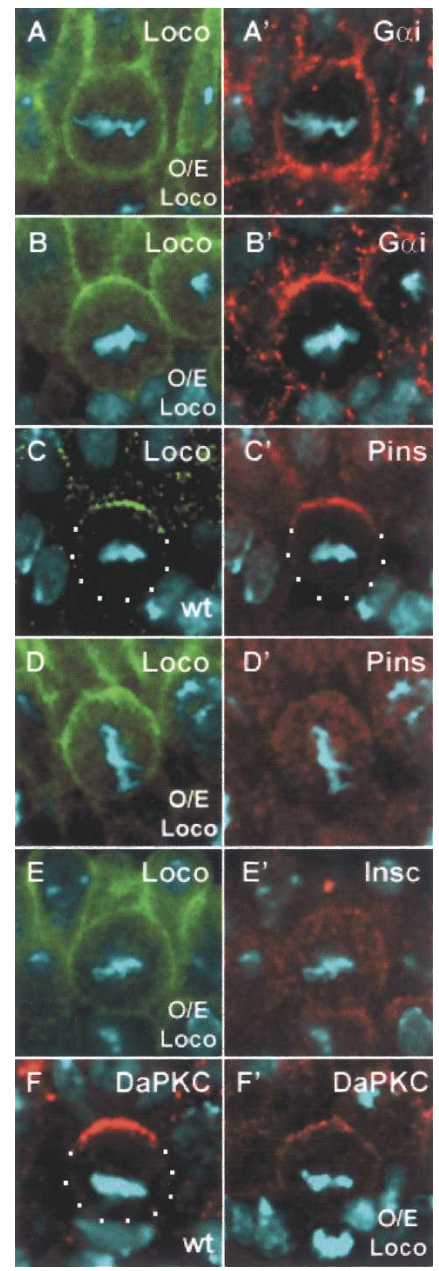

Figure 6. Ectopic expression of Loco leads to a defect in NB asymmetric divisions. Loco (green), when ectopically expressed in NBs, is localized either uniformly around the cell cortex $(A)$ or enriched at the apical cortex $(B) .\left(A^{\prime}, B^{\prime}\right)$ In both cases, Gai (red) colocalizes with ectopically expressed Loco in mitotic NBs. Ectopic Loco $(D)$, which shows much stronger intensity than that in wild-type NBs $(C)$, leads to delocalization of Pins $\left(D^{\prime}\right.$, red). In NBs ectopically expressing Loco (E, green), apical localization of Insc is also disrupted $\left(E^{\prime}\right.$, red); DaPKC (red) localizes asymmetrically but with reduced intensity $\left(F^{\prime}\right.$; wild type, F). Note that images were taken at the same gain and processed in parallel. DNA is in cyan. Apical is up.

asymmetric divisions, we overexpressed two mutant

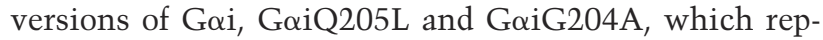
resent constitutively GTP-bound and constitutively GDP-bound forms, respectively. Previous studies suggested that overexpression of GaiQ205L perturbs SOP divisions but not NB divisions (Schaefer et al. 2001). We overexpressed GoiQ205L in wild-type NBs using the mata-ga14 VP16 V32 driver and confirmed that ectopically expressed GoiQ205L is localized primarily around the cell cortex (Fig. 7C; Schaefer et al. 2001). However, interestingly, we observed that whereas Loco remains colocalized with Goi around the cell cortex in these NBs (Fig. 7D), Pins, which normally forms an intense apical crescent in wild-type control NBs $(100 \%, n=42)$ (Fig.
$7 \mathrm{E})$, is delocalized from the apical cortex $(84 \%, n=63)$ (Fig. 7F), although a faint apical crescent can be seen occasionally. Similarly, Insc is also delocalized from the apical cortex (87\%, $n=63)$ (Fig. $7 \mathrm{H}$, wild-type control), (100\% apical, $n=42$ ) (Fig. 7G). Mira localization and segregation remain asymmetric in $100 \%$ of mitotic NBs $(n=20)$, and $2 \%$ of telophase NBs $(n=60)$ divide into similar-sized daughter cells. Delocalization of apical Pins raises the possibility that ectopically expressed GaiQ205L may preferentially bind to endogenous Loco, thereby inhibiting the Loco-mediated hydrolysis of endogenous GTP-Gai; the effect of this would be delocalization of the Pins/Insc complex at the apical cortex due to a reduction in the levels of GDP-G $\alpha$ i.

In the above situation, there should still be residual wild-type endogenous GDP-G $\alpha$ i. To create a more extreme situation, we overexpressed GaiQ205L in a Gai mutant background. Under these conditions, where

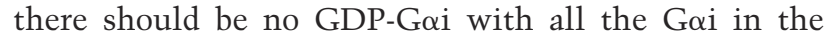
GTP-bound form, we observed more severe defects in asymmetric protein localization; the low level of Pins that can be detected is cytosolic (Fig. 7J), while Loco (Fig. 7L) and GaiQ205L (Fig. 7I,K) remain uniformly cortically localized. These observations suggest that, in vivo, Pins can associate only with GDP-G $\alpha$; GTP-G $\alpha$ i in the absence of GDP-Gai cannot direct Pins to the cell cortex; in contrast to Pins, Loco can be localized to the cortex by either GTP-G $\alpha$ i or GDP-G $\alpha$ i (see also the next paragraph). These observations along with the biochemical

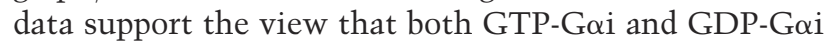
can associate with Loco in vivo, and Loco can act both as a GAP and as a GDI for G $\alpha$ i. Since G $\beta \gamma$ only binds to GDP-G $\alpha$ i, in this situation where GTP-G $\alpha$ i is in excess and GDP-G $\alpha$ i is absent, G $\beta \gamma$ will remain free and active. Indeed, under these conditions, the ability to generate daughter cell size difference is not adversely affected compared with Goi mutant NBs (data not shown) and Baz localizes asymmetrically (nonuniformly) (80\%, $n=35$ metaphase NBs) but with reduced intensity on the NB cortex (data not shown).

Although the presence of GDP-G $\alpha$ i is necessary for apical Insc/Pins/G $\alpha$ i localization, excessive GDP-G $\alpha$ i will prevent the generation of free $\mathrm{G} \beta \gamma$. For example, in

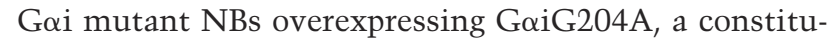
tively GDP-bound form, GaiG204A (Fig. 7M,O), Loco (Fig. 7N), and Pins (Fig. 7P) are all uniformly cortically localized; the majority of NBs divide to produce two daughter cells of similar size $(82 \%, n=57)$ (Fig. 7O,P), similar to that seen for $G \beta 13 F$ or $G \gamma 1$ mutant NBs, suggesting a failure to activate $\mathrm{G}$-protein signaling.

These data suggest that the balance between GDP-G $\alpha i$ and GTP-G $\alpha$ i is important not only to regulate G $\beta \gamma$ activity but also to asymmetrically localize Insc/Pins/ Loco.

\section{Discussion}

Previous studies have shown that heterotrimeric G-protein components play important roles in NB asymmetric divisions (Schaefer et al. 2001; Fuse et al. 2003; Yu et al. 2003; Izumi et al. 2004). In this study we consider the 
A
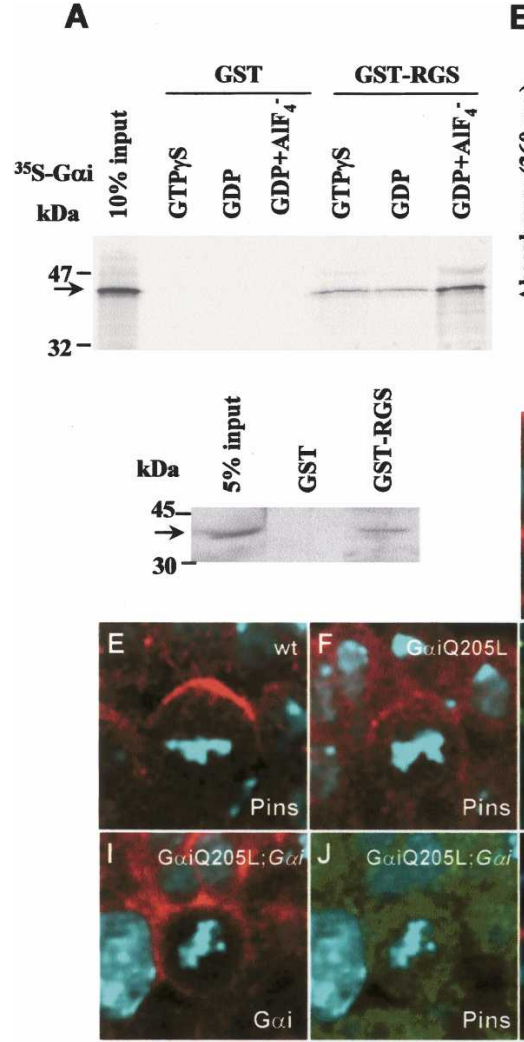

B 0.
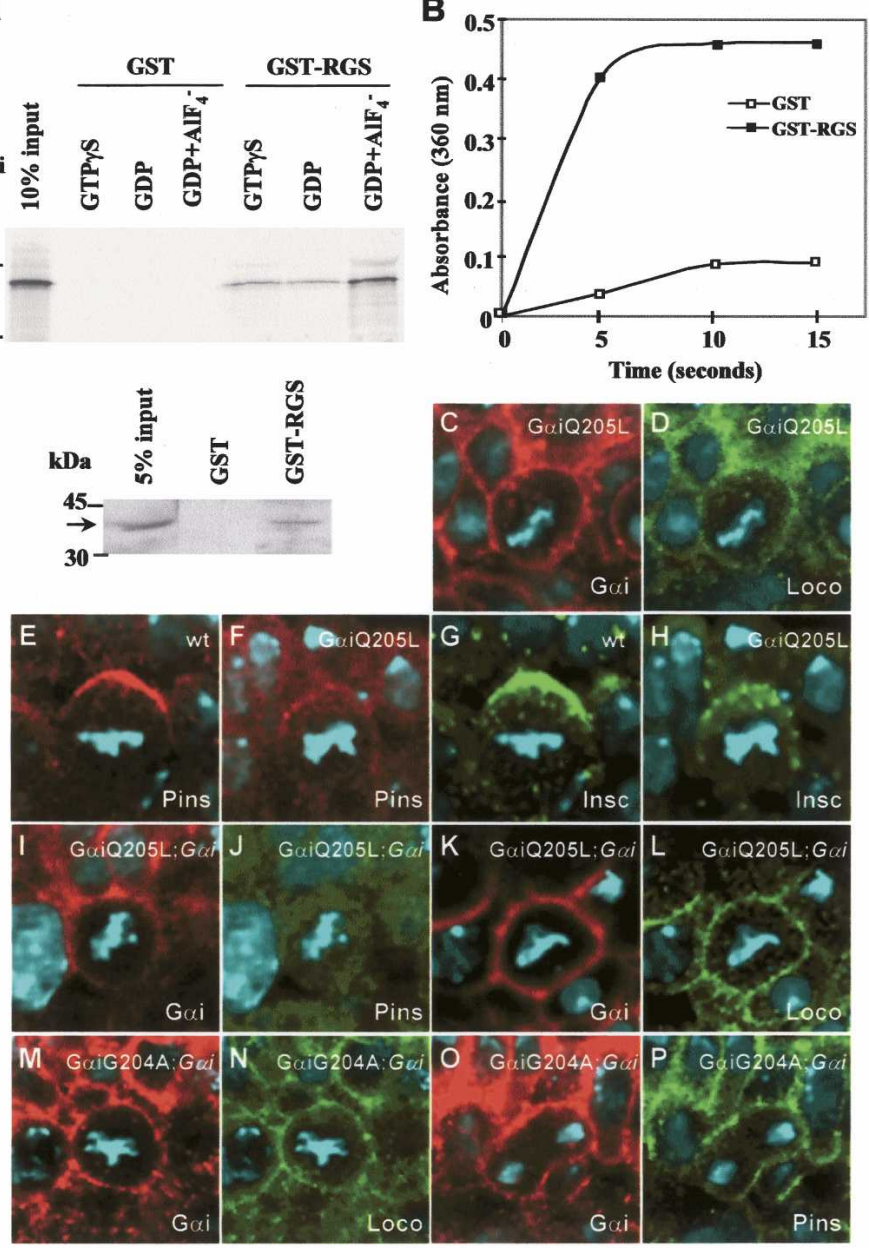

Q Receptor-independent activation of heterotrimeric $\mathbf{G}$ proteins

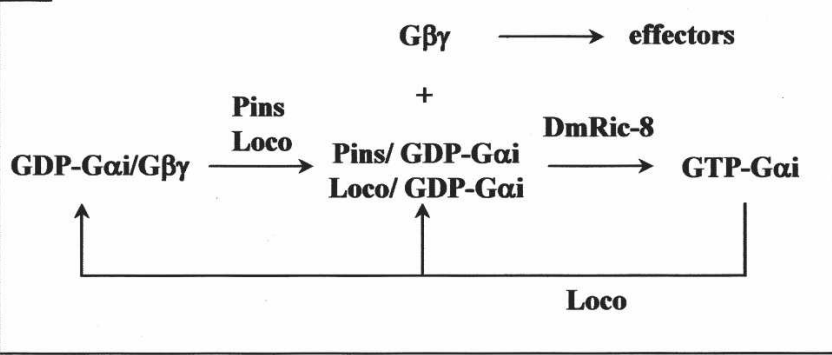

Figure 7. Loco also acts as a GAP to regulate the GTPase activity of Gai through its RGS domain. $(A)$ GST-RGS can also bind to Gai. (Upper panel) The binding assay was carried out between ${ }^{35}$ S-labeled Gai and GST alone or GST-RGS. GST-RGS has weak binding activity with G $\alpha$ i in the presence of GTP $\gamma$ S or GDP but much higher affinity to Gai in the presence of GDP and $\mathrm{AlF}_{4}{ }^{-}$. (Lower panel) GST-RGS but not GST alone is capable of complexing with endogenous Gai (see text). (B) Loco exhibits GAP activity for Gai. GST-RGS can accelerate the GTPase activity of G $\alpha$ i. $(C-P)$ Overexpression of two mutant forms of Gai in wild-type or Goi mutant backgrounds. In wild-type NBs, Pins $(E$, red) and Insc $(G$, green) are localized as intense apical crescents. In wild-type NBs ectopically expressing GaiQ205L, GaiQ205L is cortically distributed (C, red) and colocalizes with Loco ( $D$, green). $(F)$ Ectopic expression of GaiQ205L leads to disruption of Pins crescents (red) in $84 \%$ of NBs. $(H)$ Similarly, Insc localization (green) is also disrupted. Note that NBs in panels $E$ and $F$ are identical to those in panels $G$ and $H$, respectively, and those images were taken at the same gain. In Gai NBs ectopically expressing GaiQ205L $(I-L)$, GaiQ205L is cortically localized $(I, K$, red) and Pins is cytosolic $(J)$, while Loco is distributed around the cell cortex $(L)$. In Gai NBs ectopically expressing GaiG204A (the GDPbound form) $(M-P)$, GaiG204A is cortically localized during mitosis $(M, O$, red); both Loco ( $N$, green) and Pins $(P$, green) are localized around the cell cortex. (Q) A working model for receptor-independent activation of heterotrimeric G proteins in Drosophila NBs. See Discussion. issues of how heterotrimeric G-protein activation might be mediated during NB asymmetric divisions and the roles that G $\beta \gamma$, GTP-G $\alpha$ i, and GDP-G $\alpha$ i play in this process. We show that Loco is a novel asymmetrically localized component of the NB asymmetric division machinery that possesses both GDI and GAP activities for Goi. We provide evidence that indicates that the redundant GDI activities of Pins and Loco lead to the generation of free G $\beta \gamma$, which plays a crucial role for the formation of an asymmetric mitotic spindle and daughter cells of distinct size. Based on loss-of-function phenotype, G $\alpha$ i appears to play a less important role than $G \beta \gamma$ in this process; however, the proper balance between the levels of GTP- and GDP-bound forms of Gai, which may be mediated, at least in part, by the GAP activity of Loco, is crucial for the asymmetric localization of Pins and Insc. It is important to note that there may exist additional $\mathrm{G} \alpha$ subunit(s) that might functionally overlap with G $\alpha$ i in the generation of an asymmetric spindle. Therefore the possibility that G $\beta \gamma$ might mediate asymmetric spindle geometry by regulating the localization $\mathrm{G} \alpha$ subunit(s) (and GoLoco proteins) cannot be excluded at this point.

Multiple GDIs mediate receptor-independent activation of heterotrimeric $G$ proteins during $N B$ asymmetric divisions

Heterotrimeric G proteins are classically known to transmit extracellular signals to targets within the cell 
through seven transmembrane, G-protein coupled receptors (GPCRs). Upon ligand binding, GPCR acts as a GEF to stimulate release of GDP from the G $\alpha$ subunit, which, in turn, is converted to the GTP-bound form. GTP-G $\alpha$ and $\mathrm{G} \beta \gamma$ dissociate and activate their respective effectors to initiate downstream signaling. G-protein signaling is attenuated through the hydrolysis of GTP to GDP by the GTPase activity of $G \alpha$, which is accelerated by GAPs, which often contain a RGS domain. GDP-G $\alpha$ can reassociate with and inactivate $\mathrm{G} \beta \gamma$.

Analyses of loss of function of $G \beta 13 F$ and $G \gamma 1$ as well as gain of function of G $\alpha$ i in NBs have provided compelling support for the view that free G $\beta \gamma$ is required for the asymmetric localization/stability of both apical pathway components as well as the generation of asymmetric spindle and daughter cell size. Gai is required primarily for the asymmetric localization of Pins and makes only a minor contribution in regulating spindle geometry and asymmetric daughter cell size. The mechanism by which heterotrimeric G-protein activation (generation of free $G \beta \gamma$ ) is mediated in NBs has been unclear. The fact that no G-protein-coupled receptors (GPCRs) have been implicated in NB asymmetric divisions, the apparent intrinsic polarity exhibited by cultured NBs, as well as the observed GDI activity associated with Pins have raised the possibility that heterotrimeric G-protein activation may occur via a receptor-independent mechanism since GoLoco-containing molecules like Pins should be able to generate free $\mathrm{G} \beta \gamma$ from the heterotrimeric complex by competing for binding to GDP-Gai (Takesono et al. 1999; Natochin et al. 2000; Schaefer et al. 2001). However, loss of pins does not cause the majority of NBs to produce daughters of similar size and is therefore inconsistent with a failure to activate G-protein signaling.

This apparent contradiction is resolved by our observations, which indicate that receptor-independent activation of heterotrimeric G-protein signaling may be mediated through the GDI activities of both Pins and Loco. Like Pins, Loco can interact with GDP-G $\alpha$ i through its GoLoco motif and form an in vivo complex with Gai. In NBs, Loco colocalizes with G $\alpha$ i and Pins at the apical cortex throughout mitosis. Removal of maternal and zygotic loco leads to delocalization of Pins/Gai. Analysis of double mutants indicates that Loco functions redundantly with the Baz/DaPKC pathway with respect to the generation of differential daughter size. Simultaneous loss of both loco and pins results in phenotypic defects essentially indistinguishable to those seen in $G \beta 13 F$ or $G \gamma 1$ loss-of-function NBs. These observations indicate that receptor-independent activation of heterotrimeric $\mathrm{G}$ proteins during Drosophila NB asymmetric division may be achieved through the actions of the two functionally redundant GDI activities of Pins and Loco (Fig. 7Q).

The GAP activity of Loco and relevance of the

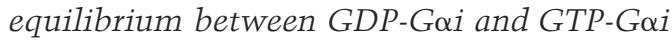

In addition to its GDI activity, Loco also possesses a RGS domain that exhibits GAP activity for Gai in vitro, suggesting that Loco can regulate Goi via two distinct modes of action, both as a GDI and as a GAP. Our studies suggest that G $\beta \gamma$, activated by the GDI activity of Pins and Loco, is crucial for NBs to produce daughters of unequal size, while the equilibrium between GDP-G $\alpha$ i and GTP-G $\alpha$ i, regulated, at least in part, by the GAP activity of Loco, is required for the localization of Insc/Pins/Loco at the apical cortex in NBs. When the equilibrium is shifted toward GTP-Goi, that is, when GaiQ205L (the constitutively GTP-bound form) is expressed in the absence of endogenous wild-type G $\alpha$ i, Pins becomes delocalized/destabilized because it requires binding to GDPGai to localize to the cell cortex; however, the ability to generate an asymmetric spindle and unequal-size daughters is not compromised since G $\beta \gamma$ function should not be compromised. Conversely, when the equilibrium is shifted toward GDP-Gai, through the ectopic expression of GoiG204A (the constitutively GDP-bound form) in the absence of endogenous wild-type G $\alpha$, free G $\beta \gamma$ fails to be generated and defects similar to those seen in $G \beta 13 F$ or $G \gamma 1$ loss of function result.

While the Loco-associated GAP activity can facilitate the conversion of GTP-G $\alpha$ i to GDP-G $\alpha$ i in NBs, how might the reverse reaction be catalyzed without invoking the involvement of a GPCR associate GEF activity? A possible nonreceptor GEF that can fulfill this role may be the Drosophila homolog of the mammalian Ric-8A (Synembrin). Mammalian Ric-8A has been shown to act as a nonreceptor GEF for $\mathrm{G} \alpha \mathrm{O}, \mathrm{Gq}$, and $\mathrm{G} \alpha \mathrm{i}_{1}$ subunits (Tall et al. 2003). Ric-8A is evolutionarily conserved from worm to mammals. More recent reports on C. elegans RIC-8 suggest that it is a GEF for the G $\alpha$ subunits, GOA-1 and GPA-16, to regulate asymmetric divisions in the zygote (Afshar et al. 2004; Couwenbergs et al. 2004; Hess et al. 2004). We also found that the fly homolog, DmRic-8, is able to associate with Gai and is involved in NB asymmetric divisions (F. Yu, unpubl.). Hence, in principle, a model along the lines schematized in Figure 7Q may explain how heterotrimeric G-protein signaling is regulated during the process of $\mathrm{NB}$ asymmetric divisions.

The role of heterotrimeric $G$ proteins in Drosophila neuroblasts and nematode zygotes

While receptor-independent activation of heterotrimeric G-protein signaling appears to be a mechanism conserved between fly and nematode, there are clear differences between the two systems. In the nematode zygote, previous studies have suggested that the $\mathrm{G} \alpha$ subunits, GOA-1 and GPA-16, are required for generation of a net pulling force from the posterior cortex that leads to the displacement of the mitotic spindle toward the posterior cortex. Either (possibly both) of the GoLoco/GPR motif proteins, GPR1/2, which are enriched at the posterior pole of the zygote (Colombo et al. 2003; Gotta et al. 2003), can act as GDIs to asymmetrically activate heterotrimeric G-protein signaling. The $\mathrm{G} \alpha$ subunits and GPR1/2 both appear to act downstream of the PAR proteins and their inactivation using RNAi results in identical spindle phenotypes that resemble those seen in 
par-2 mutants for which a reduction in cortical spindle forces have been directly demonstrated (Colombo et al. 2003; Gotta et al. 2003). More recently, it has been reported that loss of ric- 8 function also disrupts the movement of the posterior centrosome, suggesting that RIC-8 acts in the same pathway as GPR-1/2 to establish G $\alpha$ dependent force generation (Afshar et al. 2004; Couwenbergs et al. 2004; Hess et al. 2004), whereas loss of function of $r g s-7$, encoding a GAP protein for GOA-1, leads to overly vigorous posterior spindle rocking and more exaggerated size difference between two daughter cells, indicating that $\mathrm{G} \alpha$ passes through the GTP-bound state during its activity cycle to regulate the force in one-cellstage nematode embryos (Hess et al. 2004). In contrast, $\mathrm{G} \beta \gamma$ does not appear to regulate spindle displacement in the worm zygote (Srinivasan et al. 2003).

For Drosophila NBs, spindle geometry and displacement appear to be regulated to a large extent through G $\beta \gamma$ activation by the GoLoco proteins Loco and Pins. The spindle defects associated with loco/pins double loss-of-function NBs resemble those seen in the $G \beta 13 F$ and $G \gamma 1$ mutants. However, it is clear that in $G \beta 13 F$ and Gy1 mutants there is a small degree of residual asymmetry in the size of the NB daughters; this residual size difference can be removed by the additional loss of baz function (Izumi et al. 2004). There is no evidence implicating a major role for Gai in spindle asymmetry since loss of Gai has relatively mild effects (Yu et al. 2003). However, the possibility that multiple $\mathrm{G} \alpha$ subunits redundantly regulate NB spindle geometry cannot be ruled out.

Furthermore, in contrast to the C. elegans zygote where heterotrimeric G-protein signaling acts downstream of the PAR polarity cues, the precise hierarchical relationship between the heterotrimeric $\mathrm{G}$ proteins and the PAR proteins in Drosophila NBs is more complex. On the one hand, some observations can be interpreted, at least formally, to suggest that free G $\beta \gamma$ acts upstream of the apical components, since mutations in GB13F and G $\gamma 1$ cause delocalization of Pins/Loco/G $\alpha$ i and affect the stability (intensity) of the Baz and DaPKC apical crescents (Yu et al. 2003). However, reduced levels of Baz and DaPKC can nevertheless asymmetrically localize and maintain residual levels of asymmetry despite the loss of free G $\beta \gamma$, suggesting that some aspects of NB asymmetry and PAR polarity cues act in parallel or upstream of heterotrimeric G proteins (Fuse et al. 2003; Yu et al. 2003; Izumi et al. 2004). This study provides evidence that in Drosophila NBs, both Loco and Pins contribute toward the generation of free G $\beta \gamma$ and the asymmetric localization of Pins/Loco/G $\alpha$ i depends not only on G $\beta \gamma$ but also the right balance of GDP-G $\alpha$ i and GTP-G $\alpha$ i. It remains to be seen whether in NBs G $\beta \gamma$ mediates the formation of an asymmetric spindle by regulating $\mathrm{G} \alpha$ subunits.

\section{Materials and methods}

Isolation of new loco alleles

EY04589 was mobilized using $P(r y \Delta 2-3)(99 B)$ as a transposase source, and 500 independent $\mathrm{w}^{-}$revertant lines were established and analyzed. Three small deletions, $10 \mathrm{CO}^{P 237}, 10 c 0^{P 283}$, and $10 c 0^{P 452}$, that remove part or all of the loco-c1-coding region were subjected to PCR mapping and DNA sequencing to determine their precise breakpoints. The recessive lethal allele $10{ }^{P 237}$ removes the entire $10 c 0-c 1$ and loco-c2 transcripts as well as the flanking gene $m R p L 45$. The allele $10 c 0^{P 283}$ removes the region from nucleotide -310 to +2195 of the loco-c1 transcript, while 1 loco $^{P 452}$ removes the region from nucleotide -310 to +1277 of the transcript (the start point of loco-c1 transcription is +1 ). The region that is removed in the $10 c 0^{P 283}$ allele includes the RGS domain, two RBD domains, and the GoLoco motif, while $10 c 0^{P 452}$ deletes only up to and including the region encoding the RGS domain.

In $10 \mathrm{CO}^{P 283}$ mutant neuroblasts (lacking both maternal and zygotic components) overexpressing Loco-C1 (uas-loco-C1 driven with mata-Ga14 VP16 V32), Gai apical crescents can be restored in $89 \%$ of metaphase NBs $(n=74)$, and Pins crescents can been observed in $70 \%$ of metaphase NBs $(n=60)$, indicating that these defects in loco mutant NBs are due to loss of loco function. When we attempted to rescue using the same procedure with a truncated form of Loco-C1 lacking the GoLoco motif but including the RGS and RBD domains (Loco-C1 $\Delta$ GoLoco, containing amino acids 1-640), G $\alpha$ i apical crescents could be restored in $64 \%$ of mitotic neuroblasts $(n=33)$, and Pins apical crescents could be seen in $85 \%$ of neuroblasts $(n=20)$. However, in the rescue experiments with a truncated form of Loco$\mathrm{C} 1$ lacking the RGS domain (Loco-C1 $\Delta$ RGS, containing amino acids 232-830), the majority of NBs exhibit uniform cortical distribution of Pins $(81 \%, n=26)$ and Goi $(95 \%, n=23)$. Together with the biochemical experiments, these rescue results indicate that the RGS domain of Loco, and its associated GAP activity for Gai, is important for NB asymmetric divisions.

\section{Plasmid constructs, fusion proteins, and anti-Loco antibodies}

MBP-G $\alpha$ i was constructed by introducing the coding region of Gai into pMAL-c2x (NEB). Various GST fusion proteins of LocoC1 (amino acids 61-298, 337-502, 357-636, and 564-731) were generated using pGEX 4T-1 (Amersham). GST-C-Pins was generated according to Yu et al. (2002). Anti-Loco antibodies were generated in guinea pigs and affinity-purified as described in $\mathrm{Yu}$ et al. (2003). An anti-Gai antibody was raised against the full-

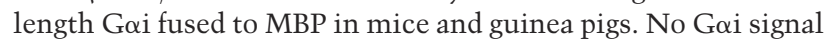
could be detected in Gai mutant embryos by Western blotting and immunofluorescent staining (data not shown), indicating

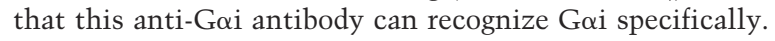

Yeast two-hybrid, protein binding assays, and GDI and GAP assays

Yeast two-hybrid assays were carried out as described in Yu et al. (2000). The fragments encoding amino acids 564-829 of Loco-C1 or amino acids $378-658$ of Pins were inserted into

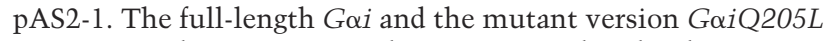
were inserted into pACT2. Their corresponding binding activities were tested based on the ability of colonies to turn blue in an X-gal filter lift assay: +, $60 \mathrm{~min} ;-$, no significant staining.

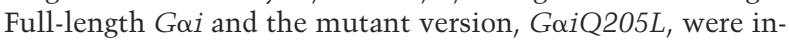
serted into pET15b (Novagene). ${ }^{35}$ S-labeled G $\alpha$ i and G $\alpha$ iQ205L proteins were produced by using TNT in vitro transcription and translation kit (Promega). The GST pull-down assays were conducted as described in Yu et al. (2000). To test for the nucleotide-dependent interaction between G $\alpha$ i and the RGS domain of Loco, $10 \mu \mathrm{L}$ of ${ }^{35} \mathrm{~S}$-labeled Gai was incubated for $30 \mathrm{~min}$ at room temperature by adding $90 \mu \mathrm{L}$ of buffer A $(50 \mathrm{mM}$ Tris- $\mathrm{HCl}$ at $\mathrm{pH}$ 8.0, $0.1 \mathrm{M} \mathrm{NaCl}, 1 \mathrm{mM} \mathrm{MgSO}_{4}, 20 \mathrm{mM}$ imidazole, $10 \mathrm{mM}$ 
mercaptoethanol, 10\% glycerol) supplemented with GTP $\gamma$ S (10 $\mu \mathrm{M})$, GDP $(10 \mu \mathrm{M})$ or GDP and $\mathrm{AlF}_{4}{ }^{-}(10$ and $30 \mu \mathrm{M})$, respectively. GST-RGS (1 $\mu$ g) or control GST ( $3 \mu \mathrm{g})$, bound to agarose beads, was separately incubated with the G $\alpha$ i mixture for 30 min at $4^{\circ} \mathrm{C}$. The agarose beads were washed four times with buffer containing the respective nucleotides and/or $\mathrm{AlF}_{4}{ }^{-}$. To test whether GST-RGS can pull down endogenous G $\alpha \mathrm{i}, 200 \mu \mathrm{g}$ of GST-RGS or GST alone was incubated with embryo extracts, followed by three washes in the lysis buffer. Bound proteins were Western-blotted with anti-Gai antibody.

$\left[{ }^{35} \mathrm{~S}\right] \mathrm{GTP} \gamma \mathrm{S}$ binding experiments were essentially performed as described in Natochin et al. (2000). Reaction mixtures containing $1 \mu \mathrm{M}$ MBP-G $\alpha$ i-GDP, $1 \mu \mathrm{M}$ GST-GoLoco (amino acids 564-731), GST-C-Pins (amino acids 378-658), or control GST were mixed with $2 \mu \mathrm{M}\left[{ }^{35} \mathrm{~S}\right] \mathrm{GTP} \gamma \mathrm{S}(1000 \mathrm{Ci} / \mathrm{mmol})$ and incubated at $30^{\circ} \mathrm{C}$ for different time periods. The reactions were terminated and measured for scintillation counts.

GTPase activity assays were performed according to the manufacturer's instructions (Enzcheck Phosphate Assay Kit; Molecular Probes). In brief, $15 \mu \mathrm{L}$ of $1 \mathrm{nmol}$ of MBP-G $\alpha$ i fusion protein was mixed with $10 \mu \mathrm{L}$ of $0.2 \mathrm{mM}$ GTP, $0.2 \mathrm{~mL}$ of 2-amino-6-mercapto-7-methylpurine ribonucleoside, 1 unit of purine nucleotide phosphorylase, and $0.78 \mathrm{~mL}$ of HEPES buffer $(\mathrm{pH} 7.5)$ and measured for the absorbance at $360 \mathrm{~nm}$. Five microliters of $1 \mathrm{M} \mathrm{MgCl}_{2}$ solution containing either GST or GSTRGS (amino acids 61-298) fusion protein was added to initiate the single turnover reaction, and the absorbance at $360 \mathrm{~nm}$ was recorded every $5 \mathrm{sec}$.

Flies, germline transformation, and RNAi experiments

Insc ${ }^{22}$, pins ${ }^{P 89}$, pins ${ }^{P 62}$, baz $^{\text {Xi106 }} \mathrm{FRT}^{9-2}$, scabrous-gal4 (scagal4), mata-gal4 VP16 V32, and UAS-Gai were described earlier in Yu et al. (2000) and Yu et al. (2003). Gß13(Ff261(FRT $T^{9-2}$ and G 1 1(N159)(FRT ${ }^{2 R-G 13}$ were kindly provided by F. Matsuzaki (Center for Developmental Biology, RIKEN, Lobe, Japan). UASGaiG204A was obtained by introducing the mutant GoiG204A cDNA in which Gly 204 had been replaced with alanine into pUAST (Brand and Perrimon 1993). Overexpression of GaiQ205L and GaiG204A in either wild-type or Gai mutant embryos was driven by mata-gal4 VP16 V32 at $26^{\circ} \mathrm{C}$. Fulllength loco-c1 (GH08607 from BDGP), loco-c1 GoLoco (encoding the region amino acids $1-630$ of the Loco-C1 protein), and loco-c1 1 RGS (encoding the region amino acids 232-830) were inserted into pUAST. The coding region of loco-c2 fused to two tandem Flag epitopes was also cloned into pUAST and hsCasper vectors and was used for germline transformation. The RNAi experiments were performed essentially as previously described in $\mathrm{Yu}$ et al. (2003).

\section{Immunocytochemistry and confocal microscopy}

Embryos were collected and fixed according to Yu et al. (2003). Rabbit anti-Asense (Y.N. Jan, University of California, San Francisco, Howard Hughes Medical Institute, CA), rabbit anti-Baz (F. Matsuzaki), rabbit anti-Insc, rabbit and mouse anti-Pins, rabbit anti-Gai (amino acids 327-355; J.A. Knoblich, Institute of Molecular Biotechnology, Vienna, Austria), guinea pig anti-Gai (this study), rabbit anti-PKC $\xi$ C20 (Santa Cruz Biotechnology), rabbit anti-G $\beta 13 F$ (F. Matsuzaki), rabbit anti-Miranda (F. Matsuzaki), rabbit anti-Pon (Y.N. Jan), rabbit anti-Numb (Y.N. Jan), mouse anti- $\alpha$-tubulin (Sigma; DM1A), rabbit anti-CNN (T.C. Kaufman, Indiana University, Howard Hughes Medical Institute, IN), anti-Pros MR1A (C.Q. Doe, University of Oregon, Howard Hughes Medical Institute, Eugene, OR), mouse anti- $\beta$ gal (Promega), Rabbit anti- $\beta$-gal (Cappel), and anti-Nrt BP106
(DSHB) were used in this study. Cy3- or fluorescein isothiocyanate (FITC)-conjugated secondary antibodies were from Jackson Laboratories. Stained embryos were incubated with ToPro-3 (Molecular Probes) to visualize DNA, and embryos were mounted in Vectashield (Vector Labs). Immunostainings were analyzed with laser scanning confocal microscope (Zeiss Meta LSM510).

\section{CoIP and Western blot}

Embryos collected from transgenic flies carrying hs-loco-c2 were heat-shocked at $34^{\circ} \mathrm{C}$ for $10 \mathrm{~min}$. Embryo extraction and CoIPs were performed as described in Yu et al. (2003). Anti-Gai or anti-Flag $(\mathrm{m} 2)$ was used for immunoprecipitation. Bound proteins were analyzed with anti-Flag, anti-Pins, and anti-Gai by Western blots (Yu et al. 2000).

\section{Acknowledgments}

We thank C.Q. Doe, Y.-N. Jan, C. Klambt, J.A. Knoblich, E. Knust, F. Matsuzaki, F. Schweisguth, H. Bellen, A. Wodarz, T. Kaufman, D. Glover, DSHB (University of Iowa), and the Bloomington stock center for generously providing antibodies and fly stocks. X.Y. is an adjunct staff, Department of Anatomy, National University of Singapore. F.Y. is supported by a Singapore Millennium Foundation Fellowship. Temasek Lifesciences Laboratory (TLL), Wellcome Trust (UK), and A ${ }^{\star}$ Star, Singapore supported this work.

\section{References}

Afshar, K., Willard, F.S., Colombo, K., Johnston, C.A., McCudden, C.R., Siderovski, D.P., and Gonczy, P. 2004. RIC-8 is required for GPR-1/2-dependent $\mathrm{G} \alpha$ function during asymmetric division of C. elegans embryos. Cell 119: 219-230.

Bellen, H.J., Levis, R.W., Liao, G., He, Y., Carlson, J.W., Tsang, G., Evans-Holm, M., Hiesinger, P.R., Schulze, K.L., Rubin, G.M., et al. 2004. The BDGP gene disruption project: Single transposon insertions associated with $40 \%$ of Drosophila genes. Genetics 167: 761-781.

Brand, A.H. and Perrimon, N. 1993. Targeted gene expression as a means of altering cell fates and generating dominant phenotypes. Development 118: 401-415.

Cai, Y., Yu, F., Lin, S., Chia, W., and Yang, X. 2003. Apical complex genes control mitotic spindle geometry and relative size of daughter cells in Drosophila neuroblast and pI asymmetric divisions. Cell 112: 51-62.

Campos-Ortega, J.A. and Hartenstein, V. 1997. The embryonic development of Drosophila melanogaster. Springer Verlag, Berlin.

Colombo, K., Grill, S.W., Kimple, R.J., Willard, F.S., Siderovski, D.P., and Gonczy, P. 2003. Translation of polarity cues into asymmetric spindle positioning in Caenorhabditis elegans embryos. Science. 300: 1957-1961.

Couwenbergs, C., Spilker, A.C., and Gotta, M. 2004. Control of embryonic spindle positioning and $\mathrm{G} \alpha$ activity by C. elegans RIC-8. Curr. Biol. 14: 1871-1876.

De Vries, L. and Gist Farquhar, M. 1999. RGS proteins: More than just GAPs for heterotrimeric G proteins. Trends Cell Biol. 9: 138-144.

Doe, C.Q. and Bowerman, B. 2001. Asymmetric cell division: Fly neuroblast meets worm zygote. Curr. Opin. Cell Biol. 13: 68-75.

Fuse, N., Hisata, K., Katzen, A.L., and Matsuzaki, F. 2003. Heterotrimeric $\mathrm{G}$ proteins regulate daughter cell size asymme- 
try in Drosophila neuroblast divisions. Curr. Biol. 13: 947954.

Giansanti, M.G., Gatti, M., and Bonaccorsi, S. 2001. The role of centrosomes and astral microtubules during asymmetric division of Drosophila neuroblasts. Development 128: 11371145.

Gotta, M. and Ahringer, J. 2001. Distinct roles for G $\alpha$ and G $\beta \gamma$ in regulating spindle position and orientation in Caenorhabditis elegans embryos. Nat. Cell Biol. 3: 297-300.

Gotta, M., Dong, Y., Peterson, Y.K., Lanier, S.M., and Ahringer, J. 2003. Asymmetrically distributed C. elegans homologs of AGS3/PINS control spindle position in the early embryo. Curr. Biol. 13: 1029-1037.

Granderath, S., Stollewerk, A., Greig, S., Goodman, C.S., O'Kane, C.J., and Klambt, C. 1999. loco encodes an RGS protein required for Drosophila glial differentiation. Development 126: 1781-1791.

Hess, H.A., Roper, J.C., Grill, S.W., and Koelle, M.R. 2004. RGS-7 completes a receptor-independent heterotrimeric G protein cycle to asymmetrically regulate mitotic spindle positioning in C. elegans. Cell 119: 209-218.

Izumi, Y., Ohta, N., Itoh-Furuya, A., Fuse, N., and Matsuzaki, F. 2004. Differential functions of $G$ protein and Baz-aPKC signaling pathways in Drosophila neuroblast asymmetric division. J. Cell Biol. 164: 729-738.

Jan, Y.N. and Jan, L.Y. 2001. Asymmetric cell division in the Drosophila nervous system. Nat. Rev. Neurosci. 2: 772-779.

Kaltschmidt, J.A. and Brand, A.H. 2002. Asymmetric cell division: Microtubule dynamics and spindle asymmetry. I. Cell Sci. 115: 2257-2264.

Kaltschmidt, J.A., Davidson, C.M., Brown, N.H., and Brand, A.H. 2000. Rotation and asymmetry of the mitotic spindle direct asymmetric cell division in the developing central nervous system. Nat. Cell Biol. 2: 7-12.

Kemphues, K. 2000. PARsing embryonic polarity. Cell 101: 345-348.

Kimple, R.J., De Vries, L., Tronchere, H., Behe, C., Morris, R.A., Gist Farquhar, M., and Siderovski, D.P. 2001. RGS12 and RGS14 GoLoco motifs are $\mathrm{G} \alpha(\mathrm{i})$ interaction sites with guanine nucleotide dissociation inhibitor Activity. J. Biol. Chem. 276: 29275-29281.

Knoblich, J.A. 2001. Asymmetric cell division during animal development. Nat. Rev. Mol. Cell. Biol. 2: 11-20.

Natochin, M., Lester, B., Peterson, Y.K., Bernard, M.L., Lanier, S.M., and Artemyev, N.O. 2000. AGS3 inhibits GDP dissociation from $\mathrm{g} \alpha$ subunits of the Gi family and rhodopsindependent activation of transducin. I. Biol. Chem. 275: 40981-40985.

Parmentier, M.L., Woods, D., Greig, S., Phan, P.G., Radovic, A., Bryant, P., and O'Kane, C.J. 2000. Rapsynoid/Partner of Inscuteable controls asymmetric division of larval neuroblasts in Drosophila. J. Neurosci. (Online) 20: RC84.

Pathirana, S., Zhao, D., and Bownes, M. 2001. The Drosophila RGS protein Loco is required for dorsal/ventral axis formation of the egg and embryo, and nurse cell dumping. Mech. Dev. 109: 137-150.

Peng, C.Y., Manning, L., Albertson, R., and Doe, C.Q. 2000. The tumour-suppressor genes $\mathrm{lgl}$ and $\mathrm{dlg}$ regulate basal protein targeting in Drosophila neuroblasts. Nature 408: 596-600.

Schaefer, M., Shevchenko, A., and Knoblich, J.A. 2000. A protein complex containing Inscuteable and the $\mathrm{G} \alpha$-binding protein Pins orients asymmetric cell divisions in Drosophila. Curr. Biol. 10: 353-362.

Schaefer, M., Petronczki, M., Dorner, D., Forte, M., and Knoblich, J.A. 2001. Heterotrimeric $\mathrm{G}$ proteins direct two modes of asymmetric cell division in the Drosophila nervous sys- tem. Cell 107: 183-194.

Schober, M., Schaefer, M., and Knoblich, J.A. 1999. Bazooka recruits Inscuteable to orient asymmetric cell divisions in Drosophila neuroblasts. Nature 402: 548-551.

Siderovski, D.P., Diverse-Pierluissi, M., and De Vries, L. 1999. The GoLoco motif: A Gai/o binding motif and potential guanine-nucleotide exchange factor. Trends Biochem. Sci. 24: 340-341.

Srinivasan, D.G., Fisk, R.M., Xu, H., and van den Heuvel, S. 2003. A complex of LIN-5 and GPR proteins regulates G protein signaling and spindle function in $C$ elegans. Genes \& Dev. 17: 1225-1239.

Takesono, A., Cismowski, M.J., Ribas, C., Bernard, M., Chung, P., Hazard, S.r., Duzic, E., and Lanier, S.M. 1999. Receptorindependent activators of heterotrimeric G-protein signaling pathways. J. Biol. Chem. 274: 33202-33205.

Tall, G.G., Krumins, A.M., and Gilman, A.G. 2003. Mammalian Ric-8A (synembryn) is a heterotrimeric $\mathrm{G} \alpha$ protein guanine nucleotide exchange factor. J. Biol. Chem. 278: 8356-8362.

Wodarz, A. and Huttner, W.B. 2003. Asymmetric cell division during neurogenesis in Drosophila and vertebrates. Mech. Dev. 120: 1297-1309.

Yu, F., Morin, X., Cai, Y., Yang, X., and Chia, W. 2000. Analysis of partner of inscuteable, a novel player of Drosophila asymmetric divisions, reveals two distinct steps in inscuteable apical localization. Cell 100: 399-409.

Yu, F., Ong, C.T., Chia, W., and Yang, X. 2002. Membrane targeting and asymmetric localization of Drosophila partner of inscuteable are discrete steps controlled by distinct regions of the protein. Mol. Cell. Biol. 22: 4230-4240.

Yu, F., Cai, Y., Kaushik, R., Yang, X., and Chia, W. 2003. Distinct roles of G $\alpha$ i and G $\beta 13 F$ subunits of the heterotrimeric G protein complex in the mediation of Drosophila neuroblast asymmetric divisions. J. Cell Biol. 162: 623-633. 


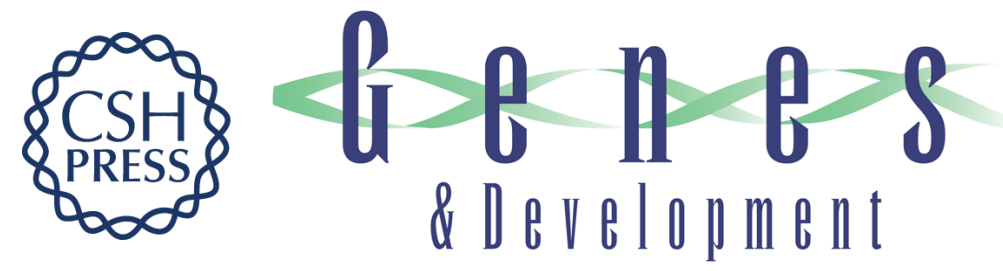

\section{Locomotion defects, together with Pins, regulates heterotrimeric G-protein signaling during Drosophila neuroblast asymmetric divisions}

Fengwei Yu, Hongyan Wang, Hongliang Qian, et al.

Genes Dev. 2005, 19:

Access the most recent version at doi:10.1101/gad.1295505

References This article cites 35 articles, 14 of which can be accessed free at:

http://genesdev.cshlp.org/content/19/11/1341.full.html\#ref-list-1

License

Email Alerting

Receive free email alerts when new articles cite this article - sign up in the box at the top

Service

right corner of the article or click here.

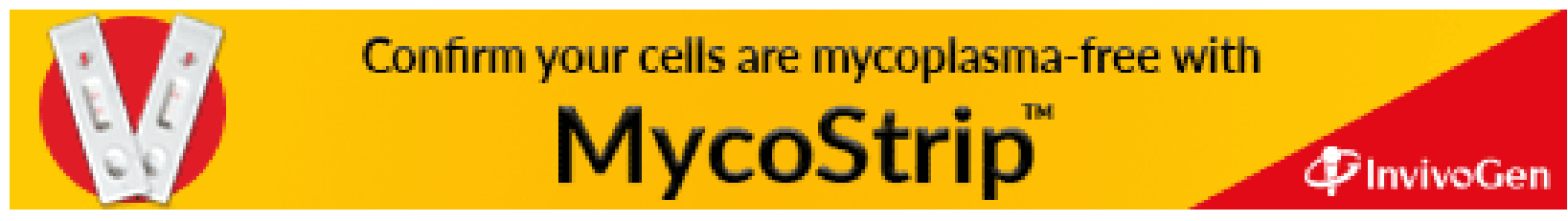

